\title{
Enhancing the mechanical properties and formability of low carbon steel with dual- phase microstructures
}

\begin{abstract}
In the present study, a special heat treatment cycle (step quenching) was used to produce a dual-phase (DP) microstructure in low carbon steel. By producing this DP microstructure, the mechanical properties of the investigated steel such as yield stress, tensile strength, and Vickers hardness were increased 14, 55, and 38\%, respectively. In order to investigate the effect of heat treatment on formability of the steel, Nakazima forming test was applied and subsequently finite element base modeling was used to predict the outcome on forming limit diagrams. The results show that the DP microstructure also has a positive effect on formability. The results of finite element simulations are in a good agreement with those obtained by the experimental test.
\end{abstract}

Keyword: Dual-phase steel; Finite element modeling; Forming limit diagram; Mechanical property; Step quenching 